\title{
Motion perception in the ageing visual system: Minimum motion, motion coherence, and speed discrimination thresholds
}

\author{
Robert J Snowden, Emma Kavanagh \\ School of Psychology, Cardiff University, Cardiff CF10 3YG, Wales, UK; e-mail: snowden@cardiff.ac.uk \\ Received 4 January 2005, in revised form 14 June 2005
}

\begin{abstract}
We aimed to address two issues: first, to describe how the perception of motion differs in elderly observers as compared to younger ones; and, second, to see if these changes in motion perception could be accounted for by the known changes in the ability of elderly observers to detect patterns (as indexed via contrast sensitivity). The lower threshold of motion, motion coherence, and speed discrimination were measured, alongside contrast sensitivity, in a group of thirty-two older (mean age 61.5 years) and thirty-two younger (mean age 23.2 years) subjects. The older observers showed losses in their ability to detect slow motions as indexed via the lower threshold of motion for random-dot patterns and for gratings of a range of spatial frequencies. They also were impaired on a test of motion coherence, but only for stimuli of a slow to medium speed, whereas faster speeds showed no decline with age. Finally, at all speeds tested the older observers required greater differences in speed in order to discriminate between patterns moving at different speeds. The pattern of losses on motion perception tasks was not predicted by the deficits of the older groups, such as loss of detection thresholds for high spatial and/or temporal frequencies. It is concluded that these hypotheses do not provide an adequate account of the data, and therefore that the losses occurring with age are complex and probably are a result of the loss of several types of cell.
\end{abstract}

\section{Introduction}

The population is steadily growing older. According to Owsley (1994), by the year 2024 a quarter of all drivers will be over 65 years of age - a statistic which raises a number of important questions. A deficit in various visual functions with increasing age has been reported in many studies (eg Elliott et al 1990; Spear 1993); however, it is still far from clear which deficits may be important in performing different visual tasks. Therefore an understanding of the changes in visual function that occur with ageing is vital if we are to successfully serve the needs of the community.

Most research into the changing visual systems of the elderly has concentrated upon measures of their static vision (ie when all elements of the scene are stationary). In this paper, we set out to systematically explore how several aspects of motion perception might be altered in the ageing visual system. We took three measures of motion perception: the minimum motion thresholds, motion coherence thresholds (for a range of speeds), and speed discrimination thresholds (again for a range of speeds). Our aim was:

(i) To describe if and how these thresholds change with the ageing process.

(ii) To see if changes in one function can explain changes in another functionie could changes in minimum motion thresholds be explained by a loss of contrast sensitivity. To this end we also took measures of spatial and temporal contrast sensitivity. However, the details of these experiments are not presented here owing to space limitations. We found that our elderly group had losses of contrast sensitivity for high

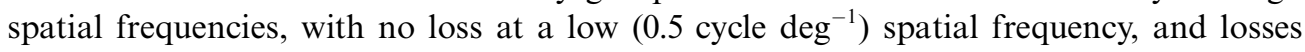
for high temporal frequencies with no loss at a low $(0.5 \mathrm{~Hz})$ temporal frequency [details are available from the first author or in Kavanagh (2003)]. 


\section{Experiment 1. Motion sensitivity - the lower threshold of motion}

There has been little previous research into motion sensitivity in the elderly population. Wood and Bullimore (1995) reported a decrease in motion sensitivity with age of the magnitude of $0.07 \mathrm{log}$ min of arc per decade. They used random-dot patterns that underwent a discrete displacement (rather than continuous motion) and reported lowest displacement thresholds.

A random-dot pattern, such as that used by Wood and Bullimore, contains many spatial frequencies. Much previous literature (eg Owsley et al 1983) has shown that sensitivity to high spatial frequencies is lost in old age (confirmed in the present population) owing to both optical and neural factors; thus, could the loss of motion sensitivity be simply due to this loss of the input of high spatial frequencies? The effects of the loss of high spatial frequencies can be mimicked by defocusing or blurring a pattern (Campbell and Green 1965), and such blurring or defocus has been shown to elevate motion thresholds. For example, Leibowitz et al (1972) used a singleline stimulus and showed that motion sensitivity was affected throughout the visual field if the stimulus was blurred. Such line stimuli also contain many spatial frequencies and it seems likely that the increase in motion thresholds was due to the loss of visibility of these high spatial frequencies. Thus any loss of motion sensitivity in old age when such stimuli are used may be simply due to losses in spatial vision rather than a loss in motion perception.

In order to control for the effects of spatial frequency, it is necessary to use a stimulus that will allow for the testing of single spatial frequencies. Sinusoidal gratings isolate a single spatial frequency, thus allowing us to extract information about the spatial and temporal properties of the motion system. However, as yet there has been little work on how the lower threshold of motion may vary with age when tested with such stimuli. Buckingham et al (1987) used a grating that underwent oscillatory motion and the observers had to discriminate this from a non-moving stimulus. The grating was presented in isolation from any other markers in the room (including the normal static window in which the grating is presented, which most experiments employ). This 'unreferenced' motion is very different from the normal measure of lower thresholds of motion used in many other studies (eg Levi et al 1984) but most valuable nonetheless. Buckingham et al (1987) found a reduced sensitivity to this motion in the older groups. Therefore, in this experiment we tested the lower threshold of motion in our older and younger participants using both random-dot patterns and sinusoidal gratings of various spatial frequencies.

\subsection{Method}

2.1.1 Observers. Sixty-four observers were used in total: thirty-two old and thirty-two young. The older group was made up of sixteen males and sixteen females with a mean age of 61.5 years $(\mathrm{SD}=4.9$ years; range $54-71$ years); the younger group contained twenty-one females and eleven males with a mean age of 23.2 years $(\mathrm{SD}=3.6$ years; range 17-30 years). Recruitment of all observers was from the local community (around Swansea, South Wales) rather than from within the university. Therefore all observers had no previous experience of psychophysical tasks and were expected to be of similar IQ and educational levels (though no formal tests were applied). All observers wore their appropriate distance correction lenses if necessary. To compensate for the lack of accommodation in the older observers, they also wore an additional lens of $+1.75 \mathrm{D}$. All observers gave their informed consent for participation in these experiments.

2.1.2 Stimuli. The stimuli were produced on a VSG 2.3 graphics board (Cambridge Research Systems) housed within a PC computer. The stimuli were displayed on a Mitsubishi Diamond Pro-20 monitor refreshed at a rate of $120 \mathrm{~Hz}$ and with an active display area of $29 \mathrm{~cm}$ by $27.5 \mathrm{~cm}$. All stimuli were viewed from a distance of $57 \mathrm{~cm}$ by binocular vision. 
The grating stimuli had a sinusoidal luminance profile, a mean luminance of $60 \mathrm{~cd} \mathrm{~m}^{-2}$, a maximum luminance $\left(L_{\max }\right)$ of $119 \mathrm{~cd} \mathrm{~m}^{-2}$, and a minimum luminance $\left(L_{\min }\right)$ of $1 \mathrm{~cd} \mathrm{~m}^{-2}$. The output of the grating generator was gamma corrected by an internal lookup table to produce accurate contrasts up to $98 \%$ contrast. The gratings were all displayed at the centre of the screen within a 'hard-edged' circular patch of $8 \mathrm{~cm}$ diameter, which from the viewing distance of $57 \mathrm{~cm}$ subtended $8 \mathrm{deg}$ at the eye. All stimuli were ramped on and off to avoid unwanted temporal transients. The contrast of the stimulus was increased linearly for the first $100 \mathrm{~ms}$, remained at full contrast for $400 \mathrm{~ms}$, and was then linearly decreased back to $\% \%$ over the final $100 \mathrm{~ms}$, making a total temporal window of $600 \mathrm{~ms}$.

The gratings were presented at spatial frequencies of $0.5,1,2$, and 4 cycles $\mathrm{deg}^{-1}$. The grating stimuli were all presented at the very supraliminal contrast of $64 \%$, so as to be clearly visible at all spatial frequencies tested.

Random-dot patterns were constructed by giving random $X$ and $Y$ coordinates to a large number of dots (4000) within a strip that notionally extended well beyond the confines of the screen. At any point in time, the visible part of the strip was $265 \mathrm{~mm}$ ( $26.5 \mathrm{deg}$ ) high by $45 \mathrm{~mm}$ ( $4.5 \mathrm{deg}$ ) wide. The density of the dots was approximately 10 dots $\mathrm{deg}^{-2}$. The active part of the screen (the background) measured $265 \mathrm{~mm}$ by $280 \mathrm{~mm}$. Which part of the strip was visible was varied randomly from trial to trial so as to give the appearance of a new random-dot pattern on each trial. Each dot measured $1 \mathrm{~mm}$ by $1 \mathrm{~mm}$ ( $0.1 \mathrm{deg}$ by $0.1 \mathrm{deg})$. Each 'dot' actually consisted of two halves whose luminance could be different so that 'subpixel' movement could be achieved (Georgeson et al 1996; Morgan and Aiba 1986) as is required for measurement of the lower threshold of motion. The luminance of the background $\left(L_{\mathrm{b}}\right)$ was $60 \mathrm{~cd} \mathrm{~m}^{-2}$ whilst the notional luminance of dots was $120 \mathrm{~cd} \mathrm{~m}^{-2}$, thus the Weber contrast $\left(\Delta L / L_{\mathrm{b}}\right)$ for the pattern was 1.0 (Moulden et al 1990). Each pattern was presented for $500 \mathrm{~ms}$ and in between presentations the screen remained at the background luminance.

2.1.3 Procedure. The same procedures were used for both the random-dot patterns and for the gratings. A two-option forced-choice procedure was used. A single pattern was presented, with the movement of the pattern (up or down) chosen randomly. The observer then reported (verbally) the direction of movement which was relayed to the computer by the experimenter. This procedure was used so as to minimise any memory load on the observer. No feedback was given as to the correctness of the response. Correct responses reduced the speed of the pattern by $1 \mathrm{~dB}$ whilst incorrect responses increased the speed by $3 \mathrm{~dB}$, thus tracking the 75\% correct level (Kaernbach 1991). Starting speed was chosen after pilot studies to be approximately $12 \mathrm{~dB}$ above the expected region of threshold. The staircase continued for 64 trials and then threshold was calculated via Probit analysis (Finney 1971). Measurements were taken in a set order for each observer commencing with the lowest to highest spatial frequency and then the random-dot pattern.

\subsection{Results}

Data from two observers (one young, one old) were not used as the former did not complete this task, whilst the thresholds of the latter were over 10 times greater (4 standard deviations) than the groups' mean.

For the random-dot patterns, the mean lower threshold of motion for the older participant group was $0.121 \mathrm{deg} \mathrm{s}^{-1}$, whilst the mean for the younger group was $0.087 \mathrm{deg} \mathrm{s}$ (plotted as the rightmost points in figure 1). An independent samples $t$-test confirmed that the older group had significantly raised thresholds $\left(t_{60}=2.32, p<0.05\right)$. As the duration of the stimulus was $500 \mathrm{~ms}$, these speeds could be expressed as displacements of 0.060 and $0.044 \mathrm{deg}$. 


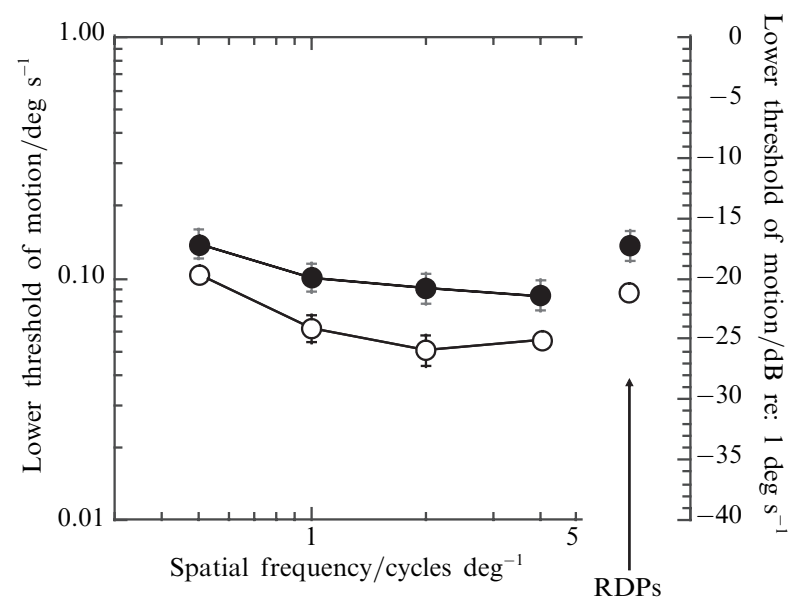

Figure 1. The lower threshold of motion plotted against the spatial frequency of the stimulus for the older group (filled symbols) and the younger group (open symbols). The left $Y$ axis shows the lower threshold of motion in $\mathrm{deg} \mathrm{s}^{-1}$, plotted logarithmically, whilst the right $Y$ axis shows the threshold in $\mathrm{dB}$ relative to a speed of $1 \mathrm{deg} \mathrm{s}^{-1}$ in linear units. The data from the experiment with random-dot patterns are shown above the notation RDP on the $X$ axis. Error bars represent \pm 1 SEM (where the error bar is not visible it is because it is smaller than the symbol size).

Inspection of figure 1 suggests that the older observers required a greater speed to achieve direction discrimination (ie a higher lower threshold of motion) at all spatial frequencies tested. These data were subjected to a three-way analysis of variance with a within-subjects factor of spatial frequency, and between-subjects factors of age and gender. There was indeed a significant main effect of age $\left(F_{1,59}=11.02, p<0.01\right)$ and a significant main effect of spatial frequency $\left(F_{3,177}=7.62, p<0.001\right)$, but no significant effect of gender $\left(F_{1,59}=0.64, \mathrm{~ns}\right)$. There was no significant interaction between age and spatial frequency $\left(F_{3,177}=1.03\right.$, ns), nor were any other interactions significant (all $F_{\mathrm{S}}<1.0$ ).

\subsection{Discussion}

The results clearly point to a significant loss of the ability to detect slow motions in the older age group. The loss was of the order of $5 \mathrm{~dB}$ (nearly doubling the speed) and appeared consistent across the stimuli used (random-dot patterns and gratings of various spatial frequencies). As such, these results confirm earlier reports of losses in the lower threshold of motion (Wood and Bullimore 1995) without the confound that the loss may be due to problems in detecting the high spatial frequencies inherent in such stimuli. Our finding of significant losses for the movement of low-spatialfrequency gratings (where we found no significant losses in contrast sensitivity) is clearly against this notion.

Whilst this result is clear in showing a loss of motion sensitivity in the older age group, it should be noted that its generality may be limited. First, though we presented our results in terms of a speed threshold (as we used continuous motion), it could also have been expressed as a displacement threshold. Of course, the older group would display the same loss when expressed in these terms, as duration was not varied in this experiment, and thus whether the loss is better expressed as one or the other is unknown. This point is more than just a pragmatic one, as quite different mechanisms may underpin motion thresholds that are governed by speed limitations and those that are governed by displacement limitations (Bonnet 1977; Boulton 1987; Exner 1888; Nakayama and Tyler 1981; Scobey and Johnson 1981; Seifert and Cavanagh 1999; Snowden 1992; Snowden and Braddick 1990), and it is possible that only one of these mechanisms may be affected in the ageing process. Further work will be needed to clarify this loss of motion 
sensitivity and its relationships to such distinctions, and, indeed, possible differences between referenced and unreferenced thresholds (Buckingham et al 1987; Levi et al 1984).

\section{Experiment 2. Motion coherence thresholds}

Whilst the lowest threshold of motion can tell us about the processing of very slow movements, by definition it does not tell us about faster motions. A technique that can be used across a range of speeds is one that is now known as motion coherence thresholds (Newsome and Paré 1988). In such experiments, a random-dot pattern is used, but only a certain percentage of the dots undergo the target motion. The other dots are either randomly repositioned on the screen or are moved at the same speed, but with a randomised direction (Scase et al 1996).

The task has become very popular, as it seems to tap into a stage of motion processing somewhat later than those with simple stimuli such as sine-wave gratings. In order to extract the direction of motion in such patterns the observer must combine information from many parts of the image, and thus it is thought to tap a global process rather than the local-motion detection of earlier stages (Morrone et al 1995; Smith et al 1994). In support of this notion, lesions to the middle temporal area of the macaque brain [an area thought to be heavily involved in the processing of motion (Snowden 1994)] produce a profound deficit for this task with relative sparing of other visual functions (Newsome and Paré 1988). In addition, imaging studies of the human brain with stimuli that differ in their level of coherence show activity in this area (amongst others) but not at the earlier stage of V1 (Braddick et al 2001).

Trick and Silverman (1991) measured motion coherence threshold in observers aged 25 to 80 years, for a very large and sparse pattern moving at $5.8 \mathrm{deg} \mathrm{s}^{-1}$. Coherence thresholds increased with age by $1.36 \%$ per decade. Wojciechowski et al (1995) also reported significantly higher motion coherence thresholds in older subjects using patterns of a very high velocity $\left(28 \mathrm{deg} \mathrm{s}^{-1}\right)$. Interestingly, this study also had a control task (differential luminance sensitivity) which did not produce any age-related effects, thus going some way to ruling out our non-specific factors (such as poor motivation, concentration, etc) as possible reasons for the results. Similar results were reported by Gilmore et al (1992) who found coherence thresholds for the older group to be nearly twice those of the younger group.

Tran et al (1998) demonstrated an increase of motion coherence thresholds with age of $0.4 \%$ per decade. However, the thresholds reported were exceedingly low (many below $2 \%$ with a 93 -year-old showing a threshold of less than $1 \%$ !) when compared to many previous studies by others reported in the literature (typically 5\%-10\%), and nearly by a factor of 10 smaller than previous reports by the same group (Silverman et al 1994; Trick and Silverman 1991). Finally, a brief report (Dengis et al 1998) also indicates a decline in motion coherence thresholds with age, with a more notable decline if the patterns are presented briefly.

Thus, there appears to be general agreement that performance on the motion coherence task deteriorates with age; however, the evidence may not be as convincing as it may at first appear. All these studies confined the measurement of motion coherence to a single speed (or displacement), though the speeds vary considerably across the studies. This may be problematic. There are some manipulations, for example of luminance and eccentricity, that alter motion coherence at some speeds but not at other speeds (eg Lankheet et al 2000). Hence the generality of this finding is not established. Given the rather mixed set of results so far reported, and the lack of a systematic look at how such variables as the speed of the motion signal might affect motion processing, we decided to examine motion coherence thresholds as a function of age. A range of speeds (dot displacements) was tested in order to establish whether deficits (if any) are equal across the spectrum of visible speeds. 


\subsection{Methods}

3.1.1 Stimuli. Stimuli were random-dot kinematograms containing 400 dots within a $9 \mathrm{~cm}$ by $9 \mathrm{~cm}$ square. Each dot was circular and had a diameter of $0.18 \mathrm{~cm}$. Dot luminance was $100 \mathrm{~cd} \mathrm{~m}^{-2}$, whilst background luminance was $20 \mathrm{~cd} \mathrm{~m}^{-2}$. Each kinematogram was displayed for $360 \mathrm{~ms}$, and consisted of 4 frames of $90 \mathrm{~ms}$. To create the kinematograms, the 400 points were randomly assigned positions within a grid of 200 by 200 pixels. Then a fixed percentage of the dots was selected randomly and the $Y$ coordinate decreased or increased by a set amount (signal dots). The remaining dots (noise dots) were assigned a random direction (but the same speed) and their $X$ and $Y$ coordinates were increased accordingly. The direction of each individual noise dot was constant across the four displacements of the stimulus. Any element that now fell outside the original area was wrapped around to the opposite side of the display. Four dot displacements were tested, namely 1,2, 4, and 8 pixel displacements per frame which in turn translate to displacements of $0.045-0.36 \mathrm{deg}(2.7-21.6 \mathrm{~min}$ of arc), and to speeds of $0.5-4.0 \mathrm{deg} \mathrm{s}^{-1}$.

3.1.2 Procedure. To establish the observer's coherence threshold, a single-interval, twooption forced-choice procedure was used. The observer was presented with a single kinematogram containing signal dots that appeared to move in an upward or downward direction. Observers were asked to indicate whether they perceived the signal dots to be moving in an upwards or downwards direction. If the observer's direction judgment was correct, the degree of coherent motion (that is the signal-to-noise ratio) was decreased by $1 \mathrm{~dB}$. Likewise, an incorrect judgment led to an increase in the degree of coherent motion of $3 \mathrm{~dB}$. For each staircase the observer completed 64 trials. To calculate a coherence threshold, the probability of a correct response at each tested displacement was calculated. Probit analysis (Finney 1971) was used to find the coherence level at which the subject had a probability of 0.75 correct. Four conditions were tested, with the signal dots moving at $0.5,1,2$, or $4 \mathrm{deg} \mathrm{s}^{-1}$. The speeds were run in a set order of slow to fast.

\subsection{Results}

Inspection of figure 2 seems to suggest a large age-related deficit in motion coherence threshold for slow speeds but not for fast speeds. The pattern of results described above was confirmed by a three-way analysis of variance (the factor of gender was included, as previous reports in the elderly had revealed gender-specific deterioration-Gilmore et al 1992). The analysis was carried out with the data expressed in logarithmic (dB), as opposed to linear (contrast), units. There was a significant main effect of the participants' age on their coherence thresholds $\left(F_{1,60}=9.67, p<0.01\right)$, and a significant main effect of stimulus speed $\left(F_{3,180}=31.06, p<0.001\right)$. A significant main effect of gender $\left(F_{1,60}=12.31, p<0.01\right)$ was also found: the males had lower thresholds $(16.6 \%)$ than women $(25.1 \%)$. The interaction between stimulus speed and participant age was also found to be significant $\left(F_{3,180}=5.07, p<0.01\right)$. There were, however, no significant interactions between stimulus speed and participant gender $\left(F_{3,180}=0.72\right.$, $p>0.05)$, or participant age and gender $\left(F_{1,60}=0.063, p>0.05\right)$. The analysis showed no significant three-way interaction between speed, age, and gender $\left(F_{3,180}=1.65\right.$, $p>0.05)$.

An independent samples $t$-test showed a significant difference between the mean coherence thresholds of the young and the old participant group at stimulus speeds of $0.5 \mathrm{deg} \mathrm{s}^{-1}\left(t_{62}=3.29, p<0.05\right)$ and $1 \mathrm{deg} \mathrm{s}^{-1}\left(t_{62}=2.75, p<0.05\right)$, but no significant difference between the performance of the young and the old groups at $2 \mathrm{deg} \mathrm{s}^{-1}$ $\left(t_{62}=0.29, p>0.05\right)$ and $4 \mathrm{deg} \mathrm{s}^{-1}\left(t_{62}=-0.17, p>0.05\right)$. 


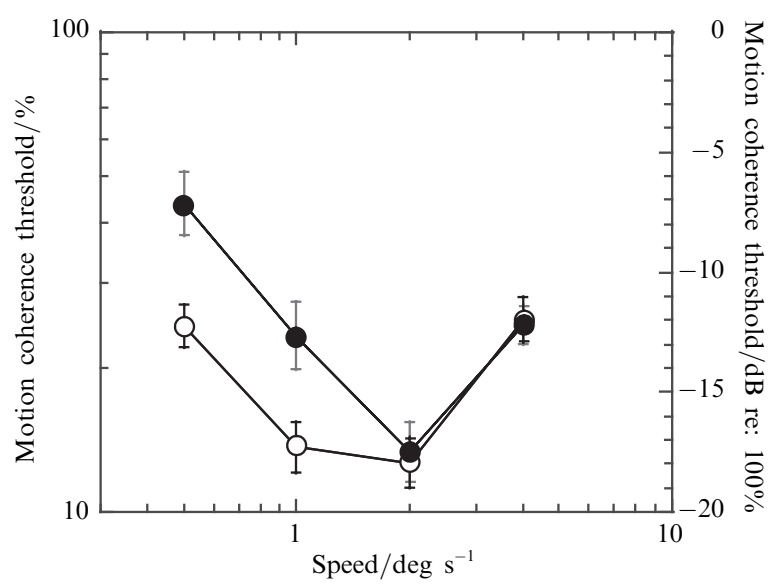

Figure 2. The motion coherence threshold plotted against the speed of the stimulus for the older group (filled symbols) and the younger group (open symbols). The left $Y$ axis shows the coherence as a percentage of the signal dots while the right $Y$ axis shows coherence in $\mathrm{dB}$ relative to all the dots acting as signal dots. Error bars represent \pm 1 SEM.

\subsection{Discussion}

Our finding that some motion coherence thresholds increase with age is in line with most previous findings (Dengis et al 1998; Gilmore et al 1992; Trick and Silverman 1991; Wojciechowski et al 1995), which all report that there is an increase in coherence thresholds with age. However, this is the first study to demonstrate that only some speeds (slow ones) are affected.

We found that the increase in thresholds with age was limited to slower speeds, a result not previously recognised in the literature owing to the simple fact that in all previous studies the tests were carried out at a single speed. This said, one might attempt to 'synthesise' this result by comparing across previous studies in which different speeds have been used. For example, the study of Wojciechowski et al reports data for a speed of $28 \mathrm{deg} \mathrm{s}^{-1}$ and clearly shows an increase in thresholds with age. This appears at first sight to contradict our findings. However, caution should be exercised. First, comparison of speed may not be the appropriate metric. Motion coherence thresholds, and, indeed, such measures as $D_{\max }$, are best described in terms of the size of the displacement per frame rather than speed of the movement per se (Baker and Braddick 1985; Braddick 1974; Snowden and Braddick 1990).

\section{Experiment 3. Speed discrimination}

Our studies so far have concentrated on the detection of moving images. Clearly another function required once the motion is detected is to be able to discriminate between motions. This could be done on the basis of direction-of-motion thresholds (eg Ball and Sekuler 1986; De Bruyn and Orban 1988) or speed discrimination thresholds (eg De Bruyn and Orban 1988; Snowden and Braddick 1991). In this paper we have chosen to explore the latter.

Once again, few studies have previously addressed age-related changes. Hills and Johnson (cited in Hills 1980) reported a study in which they examined the speedestimation abilities of older and younger drivers. They found that older drivers showed less sensitivity to vehicle speed. Scialfa et al (1987) found that the elderly judged vehicles to be travelling more quickly than did young observers. Crucially, they also reported that older adults showed reduced sensitivity to velocity differences when compared to younger observers. In a similar vein, Scialfa et al (1991) reported that the power function 
relating perceived speed to actual speed is less steep in the elderly which implies that the latter are less sensitive to speed differences.

Brown and Bowman (1987) presented ten young (aged 19 to 24 years) and ten old (aged 62 to 80 years) observers with a single spot target moving vertically at one of seven possible velocities. The observers were asked to judge whether the stimulus moved faster or slower than an implicit standard. This study failed to find any difference between speed discrimination thresholds of old and young observers. It did, however, find a significant effect of eccentricity, with speed discrimination thresholds increasing with increased eccentricity. Whilst this finding is interesting, it should be noted that testing in this study was performed under scotopic (low-light-level) conditions - thus providing little evidence about older adult performance in daylight conditions - and very small observer groups have been used. Finally, since we completed our studies, there has been a further report on this issue. Norman et al (2003) tested speed discrimination using a random-dot display imaged just into the peripheral retina. Unlike most studies on speed discrimination, in this one the two patterns to be judged were presented simultaneously rather than sequentially in time. Norman et al found a clear difference in performance between the young and old observers with thresholds deteriorating with age. This was true for all the speeds they tested $\left(1.22-24.34 \mathrm{deg} \mathrm{s}^{-1}\right)$. Why age-related deficits are found in this study, but not in the earlier study (Brown and Bowman 1987) is unclear, but several possibilities suggest themselves. First, it might be possible to judge the position of the single element presented by Brown and Bowman easily and thus supplement any speed information with this position information. The use of random-dot patterns, especially those with limited lifetimes of each element (Norman et al 2003), would eliminate such a strategy. Second, the use of a simultaneous judgment (Norman et al 2003) might introduce a shearing motion that might aid speed judgment (Snowden 1992), rather than provide speed information itself.

\subsection{Method}

Our aim was to have all methods as similar as possible to those used in the previous experiments. The following section details only the changes from previous experiments.

Gratings as described in experiment 1 were used. To measure the speed discrimination threshold, a two-interval, two-alternative forced-choice procedure was used in which the observer was presented with two gratings, one after another. Our initial aim was to investigate a wide range of baseline speeds by combining two temporal frequen-

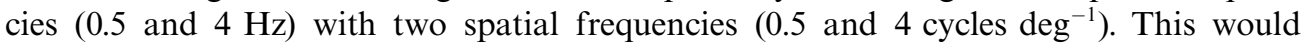
also give two combinations that both had a speed of $1 \mathrm{deg} \mathrm{s}^{-1}$ but differed in spatial and temporal frequency. However, it became apparent upon testing that the combination of 4 cycles $\mathrm{deg}^{-1}$ at $4 \mathrm{~Hz}$ produced a percept that did not 'feel' like motion but a 'blurry flicker' and judgments about its speed were difficult. The aim here was to test speed discrimination thresholds in young and old observers and so this condition was dropped, leaving three baseline speeds: $0.125 \mathrm{deg} \mathrm{s}^{-1}\left(0.5 \mathrm{~Hz}, 4\right.$ cycles $\left.\mathrm{deg}^{-1}\right), 1 \mathrm{deg} \mathrm{s}^{-1}$ $\left(0.5 \mathrm{~Hz}, 0.5\right.$ cycle deg $\left.^{-1}\right)$, and $8 \mathrm{deg} \mathrm{s}^{-1}\left(4 \mathrm{~Hz}, 0.5\right.$ cycle deg $\left.{ }^{-1}\right)$.

These speeds $\left(0.125,1\right.$, and $\left.8 \mathrm{deg} \mathrm{s}^{-1}\right)$ represent the speed $(V)$ of the standard grating. The other test grating drifted at a test speed $(V+\Delta V)$ - that is, slightly faster. The standard gratings were presented randomly in one of the two intervals with the test in the other. $\Delta V$ was varied from trial to trial via a three-up, one-down staircase, where a correct answer produced a $1 \mathrm{~dB}$ decrease and an incorrect answer a $3 \mathrm{~dB}$ increase. The observers were then given a series of practice trials (ten as a rule, but more if the observer felt they were necessary) with data collection run consisting of 64 trials. The conditions were run in a set order starting with the slowest speed. The speed discrimination threshold was calculated off-line as being the difference in speed between the two gratings $(\Delta V)$ at which the test grating was judged as being the faster in $75 \%$ of the trials. 


\subsection{Results}

Figure 3 shows the speed discrimination thresholds of the two groups of participants (younger indicated by open circles, older by filled circles) as a function of the speed of the standard stimulus. Three speeds were tested: $0.125,1$, and $8 \mathrm{deg} \mathrm{s}^{-1}$. The lefthand axis presents $\Delta V$ on a logarithmic scale in $\operatorname{deg~s}^{-1}$, whilst the right-hand axis presents $\Delta V$ in $\mathrm{dB}$ relative to a $\Delta V$ of $1 \mathrm{deg} \mathrm{s}^{-1}$ on a linear scale. As expected, $\Delta V$ rises with baseline speed and there appears to be a strong effect of age group such that the older participants have thresholds that are $3-5 \mathrm{~dB}$ greater at all speeds.

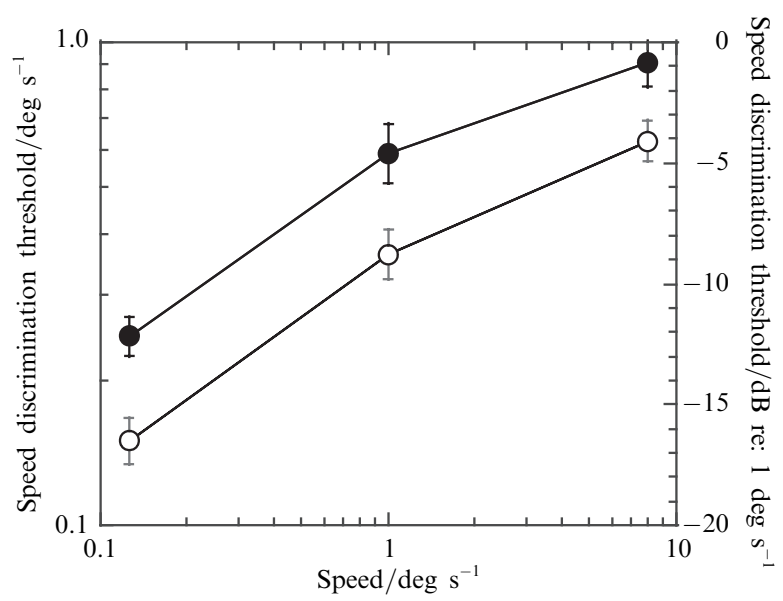

Figure 3. Speed discrimination thresholds plotted as a function of the speed of the stimulus for the older group (filled symbols) and the younger group (open symbols). The left $Y$ axis shows the speed discrimination in $\mathrm{deg} \mathrm{s}^{-1}$, plotted logarithmically, whilst the right $Y$ axis shows the threshold in $\mathrm{dB}$ relative to a speed of $1 \mathrm{deg} \mathrm{s}^{-1}$ in linear units. Error bars represent \pm 1 SEM.

An ANOVA was performed to confirm these speculations. For consistency with the previous experiments, the data used were thresholds as measured in $\mathrm{dB}$ (note that this is a logarithmic scale). Speed was treated as a within-subjects variable with three levels $\left(0.125,1\right.$, and $\left.8 \mathrm{deg} \mathrm{s}^{-1}\right)$, and age group (old, young), and gender (male, female) as between-subjects variables. There were the expected main effects of speed $\left(F_{2,110}=136.97, p<0.001\right)$, and the effect of age was also confirmed as significant $\left(F_{1,55}=20.57, p<0.001\right)$. There was also a significant main effect of gender $\left(F_{1,55}=8.84, p<0.01\right)$, such that males had lower thresholds than females. None of the two-way interactions approached significance (all $F_{\mathrm{s}}<1$ ) and the three-way interaction was not significant $\left(F_{2,110}=2.10, \mathrm{~ns}\right)$.

In figure 4 the data are presented in a manner that is common in many studies of speed perception-as a Weber percentage. On this scale, we see that Weber percentage falls with increasing speed, producing Weber percentage of around $10 \%$ at the speed of $8 \mathrm{deg} \mathrm{s}^{-1}$. The very high thresholds (Weber percentage over 100\%) are higher than previous studies have produced. However, it should be noted that these thresholds were produced for a baseline speed that was far slower than in the previous studies, and, where similar speeds are compared, thresholds are in broad agreement, given the differing stimuli and methods used. In figure 4, Weber percentage is plotted on a logarithmic scale rather than the more conventional linear scale. This was to highlight that the difference between the age groups remains constant across the speeds when expressed in this way. Of course, on a linear scale it would appear that thresholds are compromised much more at slow speeds than at high speeds. 


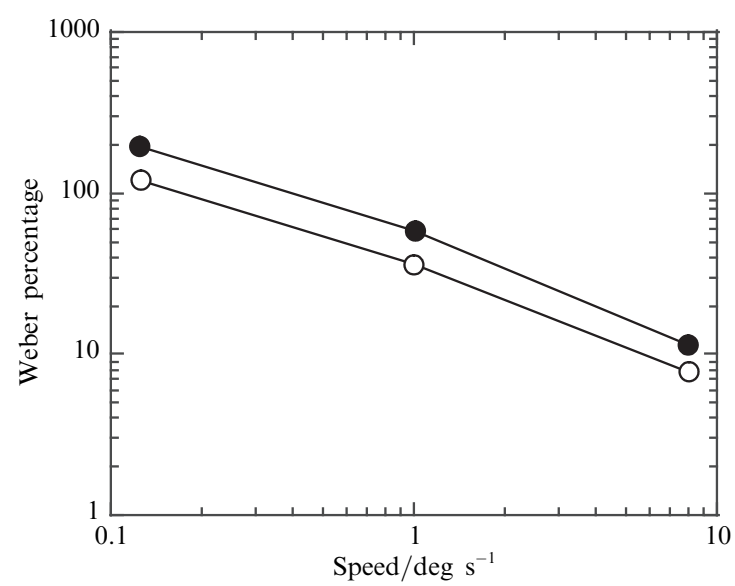

Figure 4. Speed discrimination thresholds converted to Weber percentage plotted as a function of the speed of the stimulus for the older group (filled symbols) and the younger group (open symbols) on a logarithmic axis.

\subsection{Discussion}

The results clearly show a deficit in speed discrimination associated with the ageing process. As such, our results support the findings of Norman et al (2003) in finding a deficit due to ageing that is constant across a range of speeds. Thus, the loss seems to generalise across different stimuli (dots versus gratings), presentation methods (simultaneous versus successive), and time periods (limited duration as in the present study versus unlimited presentation). Why a previous study (Brown and Bowman 1987) failed to find any difference due to age is far from clear.

\section{General discussion}

We have examined various measures of motion perception (lower threshold of motion, motion coherence thresholds, and speed discrimination thresholds). Our results show a variety of deficits namely:

(i) loss of sensitivity to visual motion at all spatial frequencies,

(ii) loss of coherence thresholds at slow movements,

(iii) loss of ability to discriminate different speeds.

However, other functions, such as sensitivity to low spatial frequencies and to low temporal frequencies, and motion coherence thresholds at medium and fast speeds, were not elevated in the aged group. Thus, a non-specific deficit (such as lack of motivation, attention, etc) is not a plausible explanation of the pattern of results obtained. The losses seen are not general but specific to certain tasks and must reflect genuine changes in the visual system of the elderly. The question of interest is whether this pattern of losses in motion perception is indeed due to problems in the motion analysing system or whether they can be explained by a faulty input into this motion analysing system.

The losses in spatial and temporal vision in the elderly have been the subject of much study. Of particular interest has been whether the losses can be accounted for by optical factors alone (which serve to reduce retinal illuminance and contrast), or whether a further loss in neural function needs to be postulated (Elliott 1987; Owsley et al 1983; Wright and Drasdo 1985). We will deliberately sidestep this issue here, and simply describe the empirical effects of these losses without recourse to their cause. We characterise the loss in sensitivity in the spatial domain as one that is absent at low spatial frequencies and increases with increasing spatial frequency. Likewise we characterise the loss in sensitivity in the temporal domain as one that is absent at low temporal frequencies and increases with increasing temporal frequency. 
Whilst just about any possible pattern of deficits can be found in the previous literature (Arden and Jacobsen 1978; Arundale 1978; Burton et al 1993; Crassini et al 1988; Derefeldt 1979; Elliott 1987; Elliott et al 1990; Kim and Mayer 1994; Kline et al 1983; Nameda et al 1989; Owsley et al 1983; Ross et al 1985; Sekuler et al 1980; Sloane and Owsley 1988; Sloane et al 1988; Tulunay-Keesey et al 1988; Wright and Drasdo 1985), the balance seems to favour the pattern we describe, though many factors (age tested, luminance of patterns, eccentricity of viewing, etc) govern the exact manifestation of the deficit. Crucially, we have measured spatial and temporal sensitivity in our population (under exactly the same conditions) and found this pattern of results. So, with this in mind we can turn to the question whether this loss of spatial and/or temporal vision can explain the results on the motion perception tasks.

\subsection{Lower threshold of motion}

Johnston and Wright (1985) found that the lower threshold of motion is invariant with contrast save for very low contrasts $(>5 \%$ ) (see also McKee et al 1986; Nakayama and Silverman 1985). As we used gratings with high contrast (64\%), even a marked loss of sensitivity (eg a log unit) would not reduce the effective contrast of such gratings into the range where contrast affects thresholds. Further, the losses of contrast sensitivity with age are confined only to high spatial frequencies, and therefore could not account for the losses in the lower threshold of motion at low spatial frequencies. The losses in contrast sensitivity at high temporal frequencies are obviously not relevant to this particular threshold which involves, by definition, very low temporal frequencies.

\subsection{Motion coherence}

The effect of change in contrast on motion coherence thresholds is minimal until very low contrasts. For example, Edwards et al (1996) have shown that alterations in the contrast of a motion coherence stimulus from $80 \%$ to $15 \%$ have no effect on thresholds (though below around 10\% there are effects - see also Trick et al 1995; van de Grind et al 1987). Given (i) the lack of any effects on motion coherence thresholds until very low contrasts, (ii) the very high contrasts used in the present study (Weber contrast of 400), and (iii) the small differences in useful contrast between the age groups, it seems highly unlikely that this could account for the results. However, we postulate a selective loss of sensitivity (and therefore effective contrast) at high spatial and high temporal frequencies. Given the broadband nature of the random-dot patterns used, we need to examine how such a 'selective loss' of high spatial or temporal frequencies can account for changes in the motion coherence threshold.

Trick et al (1995) reported that optically induced blur of $4 \mathrm{D}$ or less had no effects on coherence thresholds for a stimulus at $4.2 \mathrm{deg} \mathrm{s}^{-1}$ or $12.5 \mathrm{deg} \mathrm{s}^{-1}$. However, blur of $6 \mathrm{D}$ increased coherence thresholds for the low-contrast $(15.6 \%)$, slow-speed $\left(4.2 \mathrm{deg} \mathrm{s}^{-1}\right)$ stimulus. Furthermore, blur of $8 \mathrm{D}$ dramatically increased coherence thresholds (from $15 \%$ to $50 \%$ ) for the high-speed stimulus. Whilst these somewhat complicated findings appear on the whole to argue against loss of high-spatial-frequency information as the reason for the deficit at slow speeds found in the elderly group, they do not entirely rule this out. It would be of interest to examine motion coherence thresholds in the elderly when the spatial-frequency content of the stimulus is filtered so as to contain only specific components (Smith et al 1994).

The loss of sensitivity to high temporal frequencies seems an unlikely explanation of the results, as such a loss would be expected to be seen for the faster speeds, which was not present in the results.

\subsection{Speed discrimination}

The effect of contrast on speed discrimination (not to be confused with the effects of contrast on perceived speed-Blakemore and Snowden 1999; Snowden et al 1998; 
Thompson 1982) has been examined in a small number of studies. These studies seem to show some consistency in the finding that speed discrimination is affected by contrast only for stimuli near to contrast thresholds. Well away from this level, discrimination is unaffected by contrast level (De Bruyn and Orban 1988; Johnston et al 1999; Panish 1988; Thompson 1983).

It is also worth noting that Norman et al (2003) attempted to mimic the optical losses present in old age (Weale 1963) by the expected reduction of retinal illuminance by $0.5 \mathrm{log}$ units for their younger observers. This manipulation did not alter speed discrimination thresholds for random-dot patterns, supporting the notion that the losses cannot be accounted for by other deficits.

\subsection{Selective neuronal losses}

It is clear from the above review that the losses in motion perception we describe in this paper are not easily accounted for by the notion of a faulty input into the motion analysing system, and thus we believe that problems may occur within the motion system(s) itself.

It is generally accepted (Merigan and Maunsell 1993; Schiller and Logothetis 1990) that there are parallel visual pathways which perform somewhat different functions (Lennie 1998). Prominent amongst these pathways are those commonly termed the P- (for parvo-) and M- (for magno-) pathways. The M-stream is often thought to be highly involved in the perception of motion as it provides the greater (but not total) input into area MT-middle temporal visual area - an area thought to be intimately involved in the perception of motion (Maunsell et al 1990; Newsome et al 1989; Newsome and Paré 1988; Snowden 1994). As such, a selective (or at least greater) loss to the M-pathway (at some unspecified stage) would seem a possible candidate to explain the losses in motion perception with age. Indeed, as this hypothesis has been put forward by a number of authors to explain their particular pattern of results (eg Gilmore et al 1992; Schefrin et al 1999; Tran et al 1998), and has an historic precedence in the notion of differential changes in sustained versus transient processing (Kline 1987; Kline and Schieber 1981; Sekuler et al 1980), it deserves particular attention.

Our data are not supportive of a selective deficit in the M-pathway cells. First, such a deficit predicts a maximal loss of contrast sensitivity at low spatial and high temporal frequencies (Ansari et al 2002; Merigan and Eskin 1986; Merigan et al 1991; Merigan and Maunsell 1990) which was not the pattern seen.

Second, motion coherence thresholds should have been affected across all speeds (based upon the notion that MT lesions seem to produce a loss in motion coherence thresholds at all speeds tested-Newsome and Paré 1988); instead we got a selective deficit for slow speeds. Third, we would expect losses in speed discrimination to be greater at the faster speeds.

Finally, it is rather unclear what a deficit in the M-pathway would predict for the lower threshold of motion. Whilst some research (Lee et al 1993) has identified M-pathway cells as governing the responses to a discrete displacement of an edge, others have noted that the P-pathway cells sampling mosaic seems far more suited to motion acuity measurements (Anderson et al 1995). Hence at this stage it would seem unwise to attribute a loss in lower threshold motion as evidence for a loss of M-pathway cells (or P-pathway cells), let alone as evidence for a selective or preferential loss in either of these systems.

The above discussions do not provide good support for a selective deficit in M-pathway function. Indeed the evidence is just as supportive of a selective deficit in P-pathway cells as a loss in M-pathway cells. Others using very different techniques have also concluded that the losses are general rather than specific to one cell group (Fiorentini et al 1996). 


\subsection{Applied matters}

Whilst the neurophysiology underpinning the losses in motion perception described here and in previous work (Freeman et al 2002; Porciatti et al 1999; Willis and Anderson $2000)$ is still far from clear, their implications for everyday functions are far more transparent. We have shown that older adults have deficits in detecting the presence of low to medium fast motions, and that, when they are detected, far greater differences in speed are required for the older adults to be able to make a speed discrimination judgment. These tasks are required on a routine basis for such tasks as driving a car. As such, our evidence would suggest that routine testing of the dynamic aspects of visual function may have great benefits in ensuring the safety of all road users.

\section{References}

Anderson S J, Drasdo N, Thompson C M, 1995 "Parvocellular neurons limit motion acuity in human peripheral-vision" Proceedings of the Royal Society of London, Series B 261 129-138

Ansari E, Morgan J E, Snowden R J, 2002 "Psychophysical characterisation of early functional loss in glaucoma and ocular hypertension" British Journal of Ophthalmology 86 1131-1135

Arden G B, Jacobsen J, 1978 "A simple grating test for contrast sensitivity: Preliminary results indicate value for screening for glaucoma" Investigative Ophthalmology \& Visual Science 17 $23-32$

Arundale K, 1978 "An investigation into the variation of human contrast sensitivity with age and ocular pathology" British Journal of Ophthalmology 62 213-215

Baker C L, Braddick O J, 1982 "The basis of area and dot number effects in random dot motion perception" Vision Research $221253-1259$

Baker C L, Braddick O J, 1985 "Temporal properties of the short-range process in apparent motion" Perception $14181-192$

Ball K, Sekuler R, 1986 "Improving visual perception in older observers" Journal of Gerontology $41176-182$

Blakemore M R, Snowden R J, 1999 "The effect of contrast upon perceived speed: a general phenomenon?" Perception $2833-48$

Bonnet C, 1977 "Visual motion detection models: features and frequency filters" Perception 6 $491-500$

Boulton J C, 1987 "Two mechanisms for the detection of slow motion" Journal of the Optical Society of America A $41634-1642$

Braddick O, 1974 "A short-range process in apparent motion" Vision Research 14 519-527

Braddick O J, O’Brien J M D, Wattam-Bell J, Atkinson J, Hartley T, Turner R, 2001 "Brain areas sensitive to coherent visual motion" Perception $3061-72$

Brown B, Bowman K J, 1987 "Sensitivity to changes in size and velocity in young and elderly observers" Perception $1641-47$

Buckingham T, Whitaker D, Banford D, 1987 "Movement in decline? Oscillatory movement displacement thresholds increase with ageing" Ophthalmic and Physiological Optics $7411-413$

Burton K B, Owsley C, Sloane M E, 1993 "Aging and neural spatial contrast sensitivity: photopic vision" Vision Research $33939-946$

Campbell F W, Green D C, 1965 "Optical and retinal factors affecting visual resolution" Journal of Physiology $181576-593$

Crassini B, Brown B, Bowman K, 1988 "Age-related changes in contrast sensitivity in central and peripheral retina" Perception 17315 -332

De Bruyn B, Orban G A, 1988 "Human velocity and direction discrimination measured with random dot patterns" Vision Research $281323-1336$

Dengis C A, Sekuler A B, Bennett P J, Sekuler R, 1998 "Aging affects perceived direction of motion and detection of global flow in random dot cinematograms" Investigative Ophthalmology \& Visual Science 39 Supplement, B5046

Derefeldt G, Lennerstrand G, Lundh B, 1979 "Age variations in normal human contrast sensitivity" Acta Psychologica $57679-691$

Edwards M, Badcock D R, Nishida S, 1996 "Contrast sensitivity of the motion system" Vision Research $362411-2422$

Elliott D, 1987 "Contrast sensitivity decline with aging: A neural or optical phenomenon" Ophthalmic and Physiological Optics $7415-419$

Elliott D, Whitaker D, Macveigh D, 1990 "Neural contribution to spatiotemporal contrast sensitivity decline in healthy ageing eyes" Vision Research $30541-547$

Exner S, 1888 "Über optische Bewegungsempfindungen” Zentralblatt für Physiologie 8 437-448 
Finney D J, 1971 Probit Analysis (Cambridge: Cambridge University Press)

Fiorentini A, Porciatti V, Morrone M C, Burr D C, 1996 "Visual ageing: unspecific decline of the responses to luminance and colour" Vision Research $363557-3566$

Freeman T C A, Naji J J, Margrain T H, 2002 "Head-centred motion perception in the ageing visual system" Spatial Vision $15117-127$

Georgeson M A, Freeman T C A, Scott-Samuel N E, 1996 "Sub-pixel accuracy: Psychophysical validation of an algorithm for fine positioning and movement of dots on visual displays" Vision Research 36 605-612

Gilmore G C, Wenk H E, Naylor L A, Stuve T A, 1992 “Motion perception and aging” Psychology and Aging 7 654-660

Grind W A van de, Koenderink J J, Doorn A J van, 1987 "Influence of contrast on foveal and peripheral detection of coherent motion in moving random-dot patterns" Journal of the Optical Society of America A $41643-1652$

Hills B L, 1980 "Vision, visibility, and perception in driving" Perception 9183 - 216

Johnston A, Benton C P, Morgan M J, 1999 "Concurrent measurement of perceived speed and speed discrimination threshold using the method of single stimuli" Vision Research 39 $3849-3854$

Johnston A, Wright M J, 1985 "Lower thresholds of motion for gratings as a function of eccentricity and contrast" Vision Research $25179-185$

Kaernbach C, 1991 "Simple adaptive testing with a weighted up-down method" Perception \& Psychophysics 49227 - 229

Kavanagh E, 2003 "Motion perception and ageing", PhD thesis, Cardiff University, Cardiff, UK

Kim C B Y, Mayer M J, 1994 "Foveal flicker sensitivity in healthy aging eyes. II. Cross-sectional aging trends from 18 through 77 years of age" Journal of the Optical Society of America A 11 1958- 1969

Kline D W, 1987 "Ageing and the spatiotemporal discrimination performance of the visual system" Eye $1323-329$

Kline D W, Schieber L A, Abusmamra L A, Coyne A C, 1983 "Age, the eye, and visual channels: contrast sensitivity and response speed" Journal of Gerontology 38 (211 - 216) 323 - 329

Kline D W, Schieber F, 1981 "Visual aging: A transient/sustained shift?" Perception \& Psychophysics $29181-182$

Lankheet M J M, Doorn A J van, Bouman M A, Grind W A van de, 2000 "Motion coherence detection as a function of luminance level in human central vision" Vision Research 40 $3599-3611$

Lee B B, Wehrhahn C, Westheimer G, Kremers J, 1993 "Macaque ganglion cell responses to stimuli that elicit hyperacuity in man: detection of small displacements" Journal of Neuroscience 13 $1001-1009$

Leibowitz H W, Johnson C A, Isabelle E, 1972 "Peripheral motion detection and refractive error" Science $1771207-1208$

Lennie P, 1998 "Single units and visual cortical organization" Perception 27889 - 935

Levi D M, Klein S A, Aitsebaomo P, 1984 "Detection and discrimination of direction of motion in central and peripheral vision of normal and amblyopic observers" Vision Research $\mathbf{2 4}$ $789-800$

McKee S P, Silverman G, Nakayama K, 1986 "Precise velocity discrimination despite random variations in temporal frequency and contrast" Vision Research 26 609-619

Maunsell J H R, Nealey T A, DePriest D D, 1990 "Magnocellular and parvocellular contributions to responses in the middle temporal visual area (MT) of the macaque monkey" Journal of Neuroscience 103323 - 3334

Merigan W H, Eskin T A, 1986 "Spatio-temporal vision of macaques with severe loss of P-beta retinal ganglion cells" Vision Research 261751 - 1761

Merigan W H, Katz L M, Maunsell J H R, 1991 "The effects of parvocellular lateral geniculate lesions on the acuity and contrast sensitivity of macaque monkeys" Journal of Neuroscience $11994-1001$

Merigan W H, Maunsell J H R, 1990 "Macaque vision after magnocellular lateral geniculate lesions" Visual Neuroscience $5347-352$

Merigan W H, Maunsell J H R, 1993 “How parallel are the primate visual pathways?" Annual Review of Neuroscience $16369-402$

Morgan M J, Aiba T S, 1986 "Vernier acuity predicted from changes in the light distribution of the retinal image" Spatial Vision $1151-161$

Morrone M C, Burr D C, Viana L M, 1995 "Two stages of visual processing for radial and circular motion" Nature 376507 - 509 
Moulden B, Kingdom F, Gatley L F, 1990 "The standard deviation of luminance as a metric for contrast in random-dot images" Perception $1979-101$

Nakayama K, Silverman G H, 1985 "Detection and discrimination of sinusoidal grating displacements" Journal of the Optical Society of America A 2 267-274

Nakayama K, Tyler C W, 1981 "Psychophysical isolation of movement sensitivity by the removal of familiar position cues" Vision Research $21427-433$

Nameda N, Kawara T, Ohzu H, 1989 "Human spatio-temporal frequency performance as a function of age" Optometry and Visual Science $66760-765$

Newsome W T, Britten K H, Movshon J A, 1989 "Neuronal correlates of a perceptual decision" Nature $34152-54$

Newsome W T, Paré E B, 1988 "A selective impairment of motion perception following lesions of the middle temporal visual area (MT)" Journal of Neuroscience $82201-2211$

Norman J F, Ross H E, Hawkes L M, Long J R, 2003 "Aging and the perception of speed" Perception $3285-96$

Owsley C, 1994 "Vision and driving in the elderly" Optometry and Visual Science 71 727-735

Owsley C, Sekuler R, Siemsen D, 1983 "Contrast sensitivity throughout adulthood" Vision Research $23689-699$

Panish S C, 1988 "Velocity discrimination at constant multiples of threshold contrast" Vision Research $28193-201$

Porciatti V, Fiorentini A, Morrone M C, Burr D C, 1999 "The effects of ageing on reaction times to motion onset" Vision Research $392157-2164$

Ross J E, Clarke D D, Bron A J, 1985 "Effect of age on contrast sensitivity function: uniocular and binocular findings" British Journal of Ophthalmology 6951 - 56

Scase M O, Braddick O J, Raymond J E, 1996 "What is noise for the motion system?" Vision Research $362579-2586$

Schefrin B E, Tregear S J, Harvey L O, Werner J S, 1999 "Senescent changes in scotopic contrast sensitivity" Vision Research 393728 - 3736

Schiller P H, Logothetis N K, 1990 "The color-opponent and broad-band channels of the primate visual system" Trends in Neurosciences $13392-398$

Scialfa C T, Guzy L T, Leibowitz H W, Garvey P M, Tyrrell R A, 1991 "Age differences in estimating vehicle velocity" Psychology and Aging 6 60-66

Scialfa C T, Kline D W, Lyman B J, Kosnick W, 1987 "Age differences in judgements of vehicle velocity and distance", in Proceedings of the Annual Meeting of the Human Factors Society (New York: Human Factors Society) pp $558-561$

Scobey R P, Johnson C A, 1981 "Displacement thresholds for unidirectional and oscillatory movement" Vision Research 21 1297-1302

Seifert A E, Cavanagh P, 1999 "Position-based motion perception for color and texture stimuli: effects of contrast and speed" Vision Research $394172-4185$

Sekuler R, Hutman L P, Owsley C, 1980 "Human ageing and spatial vision" Science 2091255 - 1256

Silverman T E, Tran D B, Zimmerman K M, Feldon S E, 1994 "Dissociation between the detection and perception of motion in Alzheimer's disease" Neurology 44 1814-1818

Sloane M E, Owsley C, Jackson C A, 1988 "Aging and luminance-adaptation effects of spatial contrast sensitivity" Journal of the Optical Society of America A 5 2181-2191

Sloane M E, Owsley C, Alvarez S L, 1988 "Aging, senile miosis and spatial contrast sensitivity at low luminance" Vision Research 28 1235-1246

Smith A T, Snowden R J, Milne A B, 1994 "Is global motion really based on spatial integration of local motion signals?" Vision Research $342425-2430$

Snowden R J, 1992 "Sensitivity to relative and absolute motion" Perception $21563-568$

Snowden R J, 1994 "Motion processing in the primate cerebral cortex", in Visual Detection of Motion Eds A T Smith, R J Snowden (London: Academic Press) pp 51-83

Snowden R J, Braddick O J, 1990 "Differences in the processing of short-range apparent motion at small and large displacements" Vision Research $301211-1222$

Snowden R J, Braddick O J, 1991 "The temporal integration and resolution of velocity signals" Vision Research 31907 -914

Snowden R J, Stimpson N, Ruddle R A, 1998 "Speed perception fogs up as visibility drops" Nature 392450

Spear P D, 1993 "Neural bases of visual deficits during aging" Vision Research 33 2589-2609

Thompson P, 1982 "Perceived rate of movement depends on contrast" Vision Research 22 377-380

Thompson P, 1983 "Discrimination of moving gratings at and above detection threshold" Vision Research $231533-1538$ 
Tran D B, Silverman S E, Zimmerman K, Feldon S E, 1998 "Age-related deterioration of motion perception and detection" Graefe's Archives of Clinical and Experimental Ophthalmology 236 269-273

Trick G, Silverman S E, 1991 "Visual sensitivity to motion: Age-related changes and deficits in senile dementia of the Alzheimer type" Neurology 411437 - 1440

Trick G, Steinman S, Amyot M, 1995 "Motion perception deficits in glaucotamous optic neuropathy" Vision Research 352225 - 2233

Tulunay-Keesey U, Ver Hoeve J N, Terkla-McGrane C, 1988 "Threshold and suprathreshold spatiotemporal response throughout adulthood" Journal of the Optical Society of America A 5 $2191-2200$

Weale R A, 1963 The Aging Eye (London: H K Lewis)

Willis A, Anderson S J, 2000 "Effects of glaucoma and aging on photopic and scotopic motion perception" Investigative Ophthalmology \& Visual Science 41325 - 335

Wojciechowski R, Trick G L, Steinman S B, 1995 "Topography of the age-related decline in motion sensitivity" Optometry and Vision Science $7267-74$

Wood J M, Bullimore M A, 1995 "Changes in the lower displacement limit for motion with age" Ophthalmic and Physiological Optics 1531 - 36

Wright C E, Drasdo N, 1985 "The influence of age on the spatial and temporal contrast sensitivity function" Documenta Ophthalmologica 59385 - 395 


\section{PERCEPTION}

VOLUME 352006

www.perceptionweb.com

Conditions of use. This article may be downloaded from the Perception website for personal research by members of subscribing organisations. Authors are entitled to distribute their own article (in printed form or by e-mail) to up to 50 people. This PDF may not be placed on any website (or other online distribution system) without permission of the publisher. 